\title{
Technology-Based Pedagogical Community of Meaning (COME): Theory, Practice, and Empirical Findings
}

\author{
Shimon Azulay, Irit Wolfgor \\ Ono Academic College, Hebrew university of Jerusalem (Israel)
}

\begin{abstract}
.
This presentation has three main parts: theoretical-philosophical, pedagogical, and empirical. The first section presents a philosophical-educational theory focusing on the concept of meaning. The basic argument is that learning (like other human activities) can achieve its goal if it meets the fundamental human demand for meaning. For this purpose, a well-developed model of meaning (the Transcendence Model) is presented. One of its outcomes is the realization and manifestation of a person's life as what we call "A Player of Meaning". For the student, it refers to the pedagogical and educational sphere, which trains him for skills required nowadays.

The second section presents nine pedagogical principles for meaningful teaching. These principles are derived from the meaning model and aim to create in the classroom what we call "The Game of Meaning". Beyond meaningful learning, the principles are designed to achieve two important socio-emotional and pedagogical elements: visibility and alertness.

The third section presents an app (tchat) developed based on these principles. The teacher and the students use the app during class (currently by smartphones). The aim is to create a continuous "Game of Meaning" and turn the class into what we call "A Community of Meaning".

The presentation shows the results of a Research Study done following the use of the app among middle school students in Israel, showing a significant positive change in visibility and alertness. This change is not only a testament to the power of the app, but also to the model of meaning which underlies it.
\end{abstract}

Keywords: pedagogy, meaningful learning, visibility, alertness, community, elearning 